\title{
BMJ Open The Supporting Patient Activation in Transition to Home (sPATH) intervention: a study protocol of a randomised controlled trial using motivational interviewing to decrease re-hospitalisation for patients with COPD or heart failure
}

To cite: Flink M, Lindblad M Frykholm 0, et al. The Supporting Patient Activation in Transition to Home (SPATH) intervention: a study protocol of a randomised controlled trial using motivational interviewing to decrease re-hospitalisation for patients with COPD or heart failure. BMJ Open 2017;7:e014178. doi:10.1136/ bmjopen-2016-014178

- Prepublication history for this paper is available online. To view these files please visit the journal online (http://dx.doi. org/10.1136/bmjopen-2016014178).

Received 8 September 2016 Revised 24 May 2017 Accepted 25 May 2017

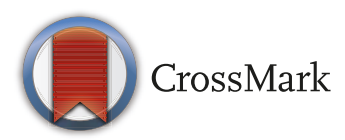

For numbered affiliations see end of article.

Correspondence to

Dr Maria Flink; maria.flink@ki.se

\section{ABSTRACT}

Introduction Deficient hospital discharging and patients struggling to handle postdischarge self-management have been identified as potential causes of re-hospitalisation rates. Despite an increased interest in interventions aiming to reduce re-hospitalisation rates, there is yet no best evidence on how to support patients in being active participants in their self-management postdischarge. The aim of this paper is to describe the study protocol for an upcoming randomised controlled trial (RCT) of the Supporting Patient in Activation to Home (SPATH) intervention.

Methods/analysis The described study is a randomised, controlled, analysis-blinded, two-site trial, with primary outcome re-hospitalisation within 90 days. In total, 290 participants aged 18 years or older with chronic obstructive pulmonary disease or congestive heart failure who are admitted to hospital and who are living in an own home will be eligible for inclusion into an intervention $(n=145)$ or control group $(n=145)$. Patients who need an interpreter to communicate in Swedish, or who have a diagnosis of dementia or cognitive impairment, will be excluded from inclusion. The SPATH intervention, developed with a theoretical base in the self-determination theory, consists of five postdischarge motivational interviewing sessions (face to face or by phone). The intervention covers the self-management areas medication management, follow-up/care plan, symptoms/signs of worsening condition and relations/contacts with healthcare providers. This RCT will add to the literature on evidence to support patient activation in postdischarge selfmanagement.

Ethics and dissemination The study is approved by the Regional Research Ethics Committee (No. 2014/149831/2) in Stockholm, Sweden. The results of the study will be published in peer-reviewed journals and presented at international and national scientific conferences.

Trial registration number NCT02823795; Pre-results.

\section{INTRODUCTION}

Hospital discharge is a hazardous period of patient care. Unwanted outcomes, such as errors in medication, therapy and in follow-up of tests and procedures, are common. ${ }^{1-3}$ Follow-up errors cause patient suffering and increased use of healthcare resources. ${ }^{4-6}$ In Sweden, one in five geriatric patients is re-hospitalised within 90 days $^{7}$ and $66 \%$ are readmitted due to the same problem causing the first hospitalisation. ${ }^{8}$ Several studies conclude that patients are unprepared for the self-management activities that follow hospitalisation. ${ }^{9-11}$ For example, between $33 \%$ and $69 \%$ of all adverse drug events postdischarge are due to patients' non-adherence to the medication list. ${ }^{12}$

A growing body of evidence indicates a beneficial effect of patient-supported interventions on the risk for 30-day re-hospitalisation. ${ }^{13}$ According to a systematic review of 42 randomised intervention trials, the most effective features of the interventions tested supported patient context and patient capacity for self-management. ${ }^{13}$ Patient activation, defined as patients' 'knowledge, skill, and confidence to manage one's health and healthcare', ${ }^{14}{ }^{15}$ has been associated with improved health outcomes and care experiences as well as reduced healthcare costs. ${ }^{14}$ Healthcare systems have much to gain regarding facilitating patient learning about self-management and engaging patients to become active partners in care ${ }^{16}$ as patients with high levels of activation have the most effective self-management skills. $^{17-19}$ 
Additionally, those with high knowledge of both their disease and its management have shown lower re-hospitalisation rates. ${ }^{20}$ Although successful results in the USA and Canada have been shown regarding interventions aiming to reduce re-hospitalisation rates, few interventions have been tested in Sweden. ${ }^{1321}$ As the healthcare system in Sweden differs from the USA and Canadian systems, it is still unclear how the results from the interventions on re-hospitalisation can be applied in a European context. Furthermore, studies on patient activation as a means to reduce re-hospitalisation and increase medication adherence have not been made in a Swedish setting.

\section{THE SUPPORTING PATIENT ACTIVATION IN TRANSITION TO HOME}

The intervention, Supporting Patient Activation in Transition to Home (sPATH), has been developed on the basis of evidence described in literature reviews, ${ }^{13} 2223$ transitional care interventions ${ }^{24}$ and our own prestudies. ${ }^{9526}$ The studies we have conducted have shown that information from nurses and physicians at discharge is distributed in parallel tracks and tailored differently to patients than to professionals in primary care, meaning that patients lost some information. ${ }^{26}$ During the discharge encounter, patients feel too stressed to grasp information, ${ }^{9}$ suggesting that support is needed to help patients make sense of the information that is provided to them. ${ }^{25}$ The systematic review and meta-analysis of Leppin et $a l^{13}$ concluded that interventions that supported patients' capacity to manage self-care activities were 1.3 times more effective than other interventions. This focus is also applied in the Care Transition Intervention ${ }^{24}$ in which medication self-management, patient-centred record, follow-up and red flags are the four pillars that support the patient to be more active during the care transition. Leppin et $a l^{13}$ and the review of Hansen et $a l^{22}$ identified that the most effective interventions included a multicomponent discharge bundle. However, Hansen et al. ${ }^{22}$ also concluded that the best evidence for reducing re-hospitalisation remained unclear.

\section{Theoretical underpinnings}

Understanding people's motivation to engage in and adhere to health promoting behaviours is of vital importance for the maintenance and improvement of people's health. Thus, the sPATH intervention is conceptually based on the self-determination theory, which is a general theory of motivation. ${ }^{27}$ The self-determination theory (SDT) recognises that people's motivation is a matter of extrinsic versus intrinsic motivation and spans over a motivational continuum. ${ }^{28}$ Behaviours can be more or less internalised, that is, autonomously regulated, ranging from external regulation (behaviour to get reward or avoid punishment) to integrated regulation (behaviour consistent with own personal goals). ${ }^{29}$ SDT has been applied to many different health-related behaviours (eg, glycaemic control, diabetes, exercise, weight loss and smoking cessation) and various clinical behaviours of healthcare practitioners (eg, counselling on smoking by physicians).

SDT incorporates a subtheory, the cognitive evaluation theory, which posits that people have three innate basic psychological needs that support the internalisation process: autonomy (the need to feel volitional and one's actions being in concordance with the sense of self), competence (the need to feel capable to achieve valued outcomes) and relatedness (the need to feel connected and close to as well as understood by others) ${ }^{29}{ }^{30}$ When these needs are met, people become more motivated and engaged in activities, as well as experience more psychological well-being. ${ }^{31}$ This, in turn predicts a positive health behaviour. ${ }^{32}$

In accordance with SDT, we designed the sPATH intervention to take into consideration how people's need for autonomy, competence and relatedness can be respected and supported by using the three specific components of autonomy support: (1) take the perspective of the patient, (2) provide the patient with choice, (3) provide rationale when choices are not possible. ${ }^{28}$ The principal of autonomy support is a core in the counselling method motivational interviewing (MI) ${ }^{33} \mathrm{MI}$ is an empirically well-tested method that has shown positive effects on health behaviour change in numerous randomised controlled trials ${ }^{34}$ for persons with chronic conditions. ${ }^{35-38}$ The 'spirit' of MI is well in alignment with SDT $^{33} 39$ and includes (1) a collaborative partnership between the patient and the healthcare provider; (2) healthcare provider evocation and activation of the patient's own motivation for change, in line with the patient's own values and needs; (3) and an honouring of patient autonomy, that is, recognition that it is the patient who makes and live with the choices. ${ }^{40}$

\section{Objectives}

The primary aim of this trial is to compare the effectiveness of the sPATH with standard care on re-hospitalisation rates within 90 days. The secondary aim is to test the effect on patient's total healthcare usage and costs, medication adherence, patient activation and experience of autonomy, competence, relatedness and quality of life at 30, 90 and 180 days postdischarge.

We hypothesise that a bridging multicomponent programme supporting patients' motivation to increased activation in self-management postdischarge (medication and care plan adherence, activation in seeking care at right level) will decrease re-hospitalisation, patient healthcare usage and costs. We also hypothesise that the intervention will increase patient activation; medication adherence; experience of autonomy, competence and relatedness; quality of life; and health-related mood.

The TIDieR checklist ${ }^{41}$ (template for intervention description and replication) has been used to inform this study protocol. 


\section{METHOD}

\section{Design and setting}

The sPATH is a randomised, controlled, analysis-blinded, ${ }^{42}$ two-site trial, set at the Karolinska University Hospital, and the Capio St. Goran Hospital, Sweden. Two departments per hospital participate in the trial: at Karolinska, the Emergency Department and the Lung Department; at Capio St. Goran, the Emergency Department and the Cardiology Department. The departments have altogether 103 beds.

\section{Eligibility criteria}

Patients aged 18 years or older with chronic obstructive pulmonary disease (COPD) or congestive heart failure admitted to hospital and who are living in their private homes will be eligible for inclusion in the trial. Exclusion criteria are diagnoses of dementia or mild cognitive impairment, need of interpreter to participate in conversations and patients with a statement of 'do-not-resuscitate' in the record. The exclusion criteria are related to the patients' possibilities to participate in the MI sessions by phone.

\section{The sPATH intervention}

Included patients transition to home will be bridged through a telephone call from a patient activation coach (medical social workers and/or nurses with education in MI and in the intervention) 2 days postdischarge. The patients will thereafter get MI sessions by the same patient activation coach with the overarching goal that the patient becomes motivated to be active in postdischarge self-management. More specifically, the goal is that the patients are motivated to acquire the knowledge, skills and confidence needed to manage the following four main activity areas: (1) medication management; (2) adhere to care plan/ follow-up visits according to the discharge plan; (3) recognise indications (symptoms/signs) that the condition is worsening and how to respond; and (4) contact and manage relations/encounters with healthcare providers. These main activity areas will be discussed in the sessions, in accordance with each patient's individual choices (table 1). Following the autonomy support of SDT, the patients may add additional areas that are crucial for them in order to handle self-management postdischarge. In all discussions, including subjects suggested by the patients, the motivation to become active or remain active during stressful situations will be targeted.

In the area medication management, patients will be encouraged to walk the patient activation coach through the medication list as a means to together explore if the patient understands the list (eg, how to read it, how to follow it, what the medications are for). Based on this, the coach will explore the patient's motivation to take control over medication management, including motivation to follow medical regimen and using a system of the patient's own choice to remember how to take medications.

In the area of care plan/follow-up, the patient will be encouraged to walk the patient activation coach through the discharge letter as a means to explore the patient's next step in the care plan (eg, does the patient have an upcoming appointment and if so, does the patient know when and why). The coach will also explore the patient's motivation to be proactive, for example, bringing discharge letters and medication lists to their specialist/ primary care follow-up or calling healthcare services when results/follow-ups are delayed.

In the area of symptoms/signs, the patients will be asked of awareness of symptoms or signs if the condition is worsening and how to respond, and the discharge letter will be explored of any information considering this. The patients' motivation to monitor his or her own status will be explored, as well as their understanding of who and when to contact if such symptoms/signs occur.

In the area of healthcare contacts, the patients will be asked to list healthcare contacts and specify why he or her has contact with this healthcare contact as a means to explore which healthcare provider they should contact for what. The area contact with healthcare providers is a thread that runs through the other three areas, that is, patients must be knowledgeable and comfortable in who to contact and when to manage medications, follow-ups and symptoms/signs. This area also includes the relatedness aspects in the self-determination theory, as patients will be encouraged to discuss communicative aspects such as what to ask for and how to best get one's voice heard.

Table 1 Overview of the Supporting Patient Activation in Transition to Home (SPATH) intervention

\section{The sPATH intervention}

\begin{tabular}{|c|c|c|c|}
\hline Point in time & Days 2-3 & Day 7 & Days 14,21 and 28 \\
\hline Setting & Postdischarge telephone call & Postdischarge encounter, face to face & $\begin{array}{l}\text { Three postdischarge telephone } \\
\text { calls }\end{array}$ \\
\hline Activity areas & $\begin{array}{l}\text { 1. Bridge the transition to } \\
\text { home by administrating acute } \\
\text { problems } \\
\text { 2. Book the postdischarge } \\
\text { encounter }\end{array}$ & $\begin{array}{l}\text { 1. Motivational interviewing to enhance } \\
\text { patients' self-management knowledge, } \\
\text { motivation and skills, that is, patients' } \\
\text { ability to manage medications, symptoms } \\
\text { to watch for and how to respond, follow- } \\
\text { up on care plans and healthcare contacts. }\end{array}$ & $\begin{array}{l}\text { 1. Continued coaching through } \\
\text { motivational interviewing to } \\
\text { improve self-management } \\
\text { knowledge, confidence and skills } \\
\text { 2. Answer any remaining } \\
\text { medication questions }\end{array}$ \\
\hline
\end{tabular}




\section{Treatment fidelity}

We are following the comprehensive treatment fidelity framework developed by the NIH Behavioral Change Consortium ${ }^{43}$ to maximise the treatment fidelity across design, provider training, delivery, receipt and enactment of treatment skills.

(1) Study design. We have explicitly used a theoretical model as a basis for the intervention. We have also determined a priori the number, length and frequency of contacts, and developed a monitoring plan to maintain consistency in dose. (2) Provider training. The coaches have received training in the intervention (written information and workshops in which the intervention and the treatment manual were discussed) and attended a 5-week MI training programme course. The course was given by members of the Motivational Interviewing Network of Trainers . (3) Treatment delivery. To enhance internal validity and ensure that the treatment is delivered as intended, all sessions between patients and coaches will be audiotaped. A random sample of audiotapes will be transmitted to a psychologist MI trainer, throughout study execution, who will audit the tapes to monitor adherence to MI principles. The MI trainer and the coaches will have monthly 1.5-hour long meetings in which feedbacks on MI adherence will be given. The MI trainer will also assist with handling encountered dilemmas/problems using MI methodology and booster sessions will be provided in the monthly meetings. A user-friendly scripted treatment manual has been developed to ensure consistency of delivery and adherence to active ingredients of the treatment. The coaching sessions will be audited by the researchers to ascertain consistency with key theoretical components of the intervention. Patient drop-out rates at each provider will be monitored and patient exit interviews will be conducted by a researcher who is independent in relation to the trial. (4) Assessment of treatment receipt is inherent in the study design and patient knowledge, skills and confidence to apply the components delivered in the intervention as well as adherence to treatment will be assessed through questionnaires and interviews. (5) Enactment of treatment skills will be assessed through a sequence of interviews with patients from both intervention and control groups.

External validity (that the treatment can be replicated and applied in real-world settings) will be assessed throughout the study period by the intervention providers' diaries and protocol as well as interviews with users and non-users.

\section{Procedures}

\section{Recruitment and sample size}

Eligible patients will be identified by one of the study's research assistants (RA) by querying registered nurses at the ward regarding potential participants two to three times a week. Once an eligible participant is identified, the RA will arrange a face-to face appointment with the patient at the hospital ward. The RA provides the patient with written information about the study together with a brief oral description regarding potential inconveniences as well as benefits with patient participation. The RA obtains written consent from willing participants regarding participation in the study. After patients have agreed to participate, they will receive an envelope containing the baseline questionnaires and instructions on how to complete them. Patients will be encouraged to complete the questionnaires before the discharge, but a stamped, addressed envelope will be provided for patients who express a desire to complete the questionnaire at home.

The recruitment target is calculated to ensure adequate statistical power to detect a difference in re-hospitalisation at 90 days, the primary endpoint, between the two groups. We estimate that the unplanned re-hospitalisation rates are $30 \%$ in the control group. To test the hypothesis that the re-hospitalisations rate is $15 \%$ lower in the intervention group than that in the control group, 242 patients (121 per group) are needed for ensure a power of $80 \%$ with the level of significance set to $5 \%$ using two-sided $X^{2}$ test. With an estimated attrition rate of $20 \%, 145$ patients per group need to be included. After 150 patients have been included, an interim analysis will be conducted by an independent statistician. The aim of the interim analysis is to decide if the estimated power calculation is adequate.

\section{Randomisation}

All eligible patients who consent to participate will be randomised to intervention or control group (145 per group). A block randomisation will be conducted to ensure equal allocation of patients in the intervention and control group. The block size will be 8 , including four interventions and four controls, as too large blocks will balance less well. The randomisation list is created by an independent statistician and is handled by the RA. After the RA has distributed the baseline data, the RA will randomise patients to either intervention or control group. The RA thereafter inform the patients, collect baseline data for patients who have completed it and send an e-notification through a secure link to the patient activation coach informing about which patients to call. Because of the nature of the intervention, it is not possible to blind patients to the treatment programme or treatment as usual; thus, there is no blinding following randomisation.

\section{Intervention group}

The patient and patient activation coach will meet in five sessions: one face to face and four telephone contacts over the course of 4 weeks (table 1). The first telephone contact takes place 2-3 days postdischarge, aiming to bridge the transition to home by addressing acute postdischarge needs such as lack of prescriptions or no show of home help services. During this telephone call, the date and time for the face-to-face encounter will be booked and the patient activation coach will encourage the patient to bring the discharge letter and medication 
list to that encounter. During the following four sessions, the patients and the coach will work together with the areas chosen by the patient. The first of these sessions (7 days postdischarge) will be a face-to-face meeting at a meeting room in the hospital. Patients will be offered to have their transportation to the hospital arranged by the coach. For patients unwilling or unable to visit the hospital, the session will either be held by phone or a home visit will be arranged. The following three sessions (14, 21 and 28 days postdischarge) will be held by phone. The scheduled days are to be considered as guiding principles. The patient and the coach will book days that fit with the patient's preferences and needs.

\section{Control group}

Patients in the control group will receive standard care. This includes the standard follow-up procedures as decided upon discharge, for example, follow-up of medical needs at the primary healthcare centre or a specialist or follow-up of social services needs by a community social worker. Patients with COPD and heart failure have either a healthcare contact at a specialist department at the hospital or, when the disease is not considered as too severe, a healthcare contact at the primary healthcare centre. Patients who are discharged from an emergency ward do not by standard receive any counselling or support to manage their self-management postdischarge needs or motivate to activation.

\section{Data collection}

All patient-reported outcome measures, PROMs (see details below and table 2), will be gathered from both intervention and control group at baseline, at the end of the fourth week of intervention (30 days after randomisation), at a 2-month follow-up (90 days after randomisation) and at a 5-month follow-up (180 days after randomisation). At baseline, patients will be asked to complete a questionnaire with sociodemographic data (eg, education, income, marital status) in addition to the PROMs, and an RA will collect these questionnaires before patient discharge. Patients will at the same time be given a stamped, addressed envelope, including the Care

Table 2 Timeline and overview over the study

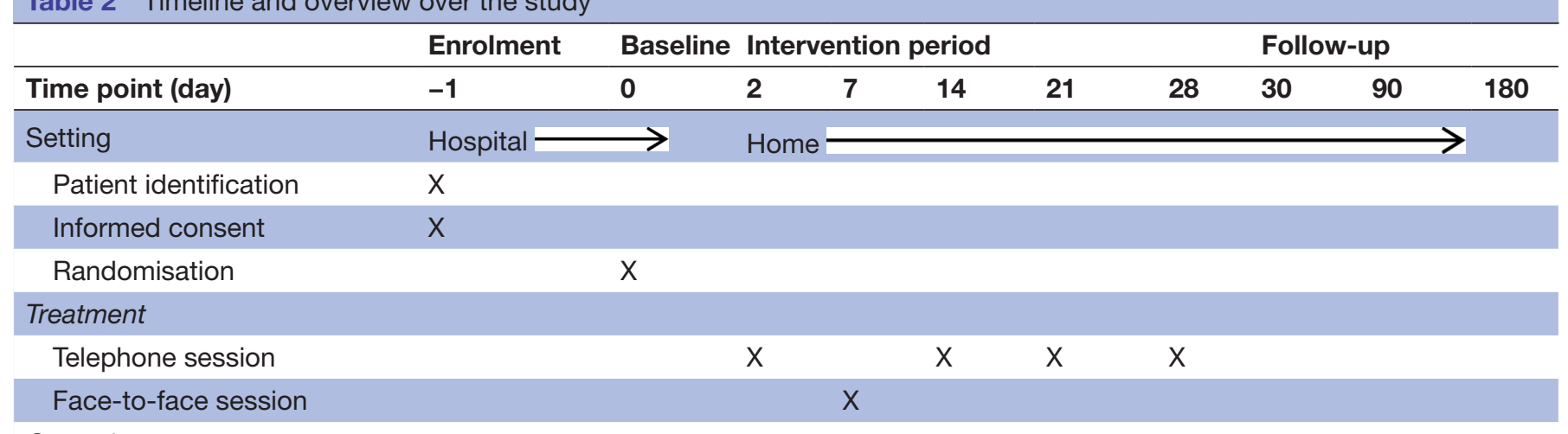

Control

Standard care

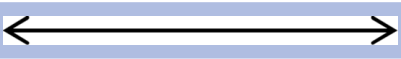

Primary outcome

Re-hospitalisation rate*

Secondary outcomes

Demographic measures

CTM-3

$X$

PAM-13

$x$

\begin{tabular}{llrr} 
PAM-13 & $X$ & $X$ & $X$ \\
EQ-5D-5L & $X$ & $X$ & $X$ \\
BPNSFS & $X$ & $X$ & $X$ \\
MMAS-8 & $X$ & $X$ & $X$ \\
PHQ-9 & $X$ & $X$ \\
Medication adherence† & & $X$ & $X$ \\
Total healthcare usagef & & $X$ & $X$ \\
\hline
\end{tabular}

${ }^{*}$ Register data on re-hospitalisations will be retrieved from the Register for Healthcare Encounters.

†Data on medication usage will be retrieved from the Prescribed Drug Registry.

$\ddagger$ Total healthcare usage (including re-hospitalisation, emergency room and primary care visits) will be retrieved for the year before inclusion in the study, from the Register for Healthcare Encounters.

BPNSFS, Basic Psychological Need Satisfaction and Frustration Scale; CTM-3, Care Transition Measure (three item); EQ-5D-5L,European Quality of Life-5 Dimensions 5 Levels; MMAS-8, Morisky Medication Adherence Scale; PAM-13, Patient Activation Measure; PHQ-9, Patient Health Questionnaire. 
Transition Measure, CTM- $3,{ }^{44}$ to be completed within 1 week and sent back to the research group. The CTM-3 is a three-item questionnaire on patient perspectives on co-ordination of hospital discharge care, where responses are given to one of five available alternatives. Timeline and an overview of the outcome measures are given in table 2.

\section{Primary outcome}

Re-hospitalisation rate is used as the primary outcome, and will be retrieved from the Register for Healthcare Encounters, at 90 days after inclusion.

\section{Secondary outcomes}

Patient activation will be measured using the Patient Activation Measure $13 .{ }^{15}{ }^{45}$ It is used for patient self-report on knowledge, skills and confidence for self-management of one's health. It consists of 13 questions, where responses can be given on one of five available alternatives.

Health-related quality of life will be measured using European Quality of Life-5 Dimensions 5 Levels (EQ-5D-5L), ${ }^{46}$ which is a general and standardised instrument for measuring health outcome. It consists of five questions/ dimensions on health status (mobility, self-care, usual activities, pain/discomfort, anxiety/depression), each of which can take one of five responses/levels. It is complemented with one question on perceived overall health status, where a response is given using visual analogue scale of $0-100$.

Basic Psychological Need Satisfaction and Frustration Scale 18 item ${ }^{47}$ will be used to assess the individual's experience of autonomy, competence and relatedness, which are conditions posited to foster more intrinsic forms of motivation and engagement for activities.

Depression will be assessed using the Patient Health Questionnaire, PHQ-9, which is widely applied to assess depression in primary healthcare. ${ }^{48}$ The PHQ-9 is a nineitem depression module derived from the full PHQ, where responses are given on one of four alternatives. The items come directly from the nine signs and symptoms of major depression delineated in the Diagnostic and Statistical Manual of Mental Disorders, Fourth Edition. ${ }^{49}$

Medication adherence will be calculated using data from the Prescribed Drug Registry and the results from the Morisky Medication Adherence Scale $8 .^{50}$ It consists of eight items on medication routines and intake, with responses given either as yes/no (only the last question offers a choice of five alternatives).

The cost-effectiveness of the study will be measured as total healthcare usage and intervention costs (education, devices, personnel). The net cost will be compared with the gains in quality-adjusted life-years (QALY), and the cost per QALY gained will be estimated. Healthcare usage (including emergency room and primary care visits) will be retrieved from the Register for Healthcare Encounters, the year before inclusion in the study and at 30, 90 and 180 days after inclusion.

\section{Statistical analyses}

Analyses of primary and secondary outcomes will be conducted on an intention-to-treat basis, so all included patients will be analysed as randomised. Generalised linear mixed models ${ }^{51}$ (GLMMs) will be used for analyses of between group differences when all measurements are considered. The GLMM will be adjusted for potential confounders (eg, demographic variables). Potential correlations between outcome measures and factors such as gender, age and social economic status will be examined. $\chi^{2}$ and independent $t$ tests will be used to assess crude potential group differences, and statistically significant variables will be included in the final multiple model. The $\chi^{2}$ test is used to assess the differences between groups when variables are categorical, and the McNemar test is used when testing within-group changes over time. The independent sample $t$ test will be used to assess differences in means between study groups for continuous variables with normal distribution. All tests will be two sided. Overall, p values $<0.05$ will be considered statistically significant.

\section{Ethical considerations}

Ethical considerations will be continuously taken into account throughout the research process and the study is approved by the Regional Research Ethics Committee (Dnr 2014/1498-31/2).

The study will be conducted in line with the concerns raised by the Helsinki Declaration ${ }^{52}$ in relation to dignity and integrity for participants in a research project. Hence, participants will be provided with adequate amount of time to consider their participation and will be given an opportunity to ask questions. The participants will also be informed that participation is voluntary, as well as being informed of their right to withdraw from the study as long as data have not been analysed, without any prejudice to future medical treatment. ${ }^{53}$ All study-related information regarding participants will be stored in a secure cabinet in accordance with $\mathrm{WMA}^{52}$ and ALLEA $^{53}$ descriptions. To maintain participant confidentiality, all reports, data collection, process and administrative forms, together with consent forms, will be identified by a number code, in line with ALLEA. ${ }^{49}$

\section{Trial status}

The intervention recruited the first patients in September 2016, and we expect recruitment to be complete in September 2017. The expected completion date of the project, including all follow-up appointments, is January 2018.

\section{Author affiliations}

${ }^{1}$ Department of Learning, Informatics, Management, and Ethics, Karolinska Institutet, Stockholm, Sweden

${ }^{2}$ Department of Social Work, Karolinska University Hospital, Stockholm, Sweden

${ }^{3}$ School of technology and Health, KTH, Royal Institute of technology, Stockholm, Sweden

${ }^{4}$ Department of Medical and Health Sciences, Division of Community Medicine, Linköping University, Linköping, Sweden 
${ }^{5}$ School of Health and Caring Sciences, Faculty of Health and Life Sciences Linnaeus University, Kalmar, Sweden

${ }^{6}$ Kalmar County Hospital, Kalmar, Sweden

Contributors Study design: MF, ML, PN, KÅ, ME. Writing of draft manuscript: MF, OF, ÅK, ME. Revision of draft/finalising manuscript: MF, ML, OF, ÅK, PN, KÅ, ME.

Competing interests None declared.

Ethics approval Regional Research Ethics Committee (No. 2014/1498-31/2) in Stockholm, Sweden.

Provenance and peer review Not commissioned; externally peer reviewed.

Data sharing statement № additional data are available.

Open Access This is an Open Access article distributed in accordance with the Creative Commons Attribution Non Commercial (CC BY-NC 4.0) license, which permits others to distribute, remix, adapt, build upon this work non-commercially, and license their derivative works on different terms, provided the original work is properly cited and the use is non-commercial. See: http://creativecommons.org/ licenses/by-nc/4.0/

(c) Article author(s) (or their employer(s) unless otherwise stated in the text of the article) 2017. All rights reserved. No commercial use is permitted unless otherwise expressly granted.

\section{REFERENCES}

1. Arora VM, Prochaska ML, Farnan JM, et al. Problems after discharge and understanding of communication with their primary care physicians among hospitalized seniors: a mixed methods study. $J$ Hosp Med 2010;5:385-91.

2. Forster AJ, Murff HJ, Peterson JF, et al. The incidence and severity of adverse events affecting patients after discharge from the hospital. Ann Intern Med 2003;138:161-7.

3. Moore C, Wisnivesky J, Williams S, et al. Medical errors related to discontinuity of care from an inpatient to an outpatient setting. J Gen Intern Med 2003;18:646-51.

4. Jencks SF, Williams MV, Coleman EA. Rehospitalizations among patients in the medicare fee-for-service program. $N$ Engl $\mathrm{J} \mathrm{Med}$ 2009;360:1418-28.

5. Oduyebo I, Lehmann CU, Pollack CE, et al. Association of selfreported hospital discharge handoffs with 30-day readmissions. JAMA Intern Med 2013;173:624-9.

6. Vashi AA, Fox JP, Carr BG, et al. Use of hospital-based acute care among patients recently discharged from the hospital. JAMA 2013;309:364-71.

7. Stäck P, Forsberg B, Högberg M, et al. [The risk of acute readmission can be predicted. former care consumption patterns and certain diagnoses are strongly predictive]. Lakartidningen 2012;109:2211-5.

8. Sveriges Kommuner och landsting. fyra områden för att undvika onödiga sjukhusvistelser. Linköping, 2013.

9. Flink M. Patients' position in care transitions - an analysis of patient participation and patient-centeredness. Karolinska Institutet 2014.

10. Fuji KT, Abbott AA, Norris JF. Exploring care transitions from patient, caregiver, and health-care provider perspectives. Clin Nurs Res 2013;22:258-74.

11. Wagner EH, Austin BT, Davis $\mathrm{C}$, et al. Improving chronic illness care: translating evidence into action. Health Aff 2001;20:64-78.

12. Mulhem E, Lick D, Varughese $\mathrm{J}$, et al. Adherence to medications after hospital discharge in the elderly. Int J Family Med 2013;2013:1-6.

13. Leppin AL, Gionfriddo MR, Kessler M, et al. Preventing 30-day hospital readmissions: a systematic review and meta-analysis of randomized trials. JAMA Intern Med 2014;174:1095-107.

14. Hibbard JH, Greene J. What the evidence shows about patient activation: better health outcomes and care experiences; fewer data on costs. Health Aff 2013;32:207-14.

15. Hibbard JH, Stockard J, Mahoney ER, et al. Development of the Patient Activation Measure (PAM): conceptualizing and measuring activation in patients and consumers. Health Serv Res 2004;39(4 Pt 1):1005-26.

16. Barlow J, Wright C, Sheasby J, et al. Self-management approaches for people with chronic conditions: a review. Patient Educ Couns 2002;48:177-87.

17. Begum N, Donald M, Ozolins IZ, et al. Hospital admissions, emergency department utilisation and patient activation for selfmanagement among people with diabetes. Diabetes Res Clin Pract 2011;93:260-7.
18. Mitchell SE, Gardiner PM, Sadikova E, et al. Patient activation and 30-day post-discharge hospital utilization. J Gen Intern Med 2014;29:349-55.

19. Shively MJ, Gardetto NJ, Kodiath MF, et al. Effect of patient activation on self-management in patients with heart failure. $J$ Cardiovasc Nurs 2013;28:20-34.

20. Kommuri NV, Johnson ML, Koelling TM. Relationship between improvements in heart failure patient disease specific knowledge and clinical events as part of a randomized controlled trial. Patient Educ Couns 2012;86:233-8.

21. Reinius P, Johansson M, Fjellner A, et al. A telephone-based case-management intervention reduces healthcare utilization for frequent emergency department visitors. Eur J Emerg Med 2013;20:327-34.

22. Hansen LO, Young RS, Hinami K, et al. Interventions to reduce 30-day rehospitalization: a systematic review. Ann Intern Med 2011:155:520-8.

23. Hesselink G, Zegers M, Vernooij-Dassen M, et al. Improving patient discharge and reducing hospital readmissions by using intervention mapping. BMC Health Serv Res 2014;14:389.

24. Coleman EA, Parry C, Chalmers S, et al. The care transitions intervention: results of a randomized controlled trial. Arch Intern Med 2006;166:1822-8.

25. Flink M, Ekstedt M. Preparing patients for self-management at home - an observational case study of the hospital discharge process. Submitted to BMJ Quality and Safety.

26. Wibe T, Ekstedt M, Hellesø R. Information practices of health care professionals related to patient discharge from hospital. Inform Health Soc Care 2015;40:198-209.

27. Ryan RM, Deci EL. Self-determination theory and the facilitation of intrinsic motivation, social development, and well-being. Am Psychol 2000;55:68-78.

28. Sheldon KM, Williams G, Joiner T. Self-determination theory in the clinic. motivating physical and mental health. New Haven: Yale University Press, 2003

29. Patrick H, Williams GC. Self-determination theory: its application to health behavior and complementarity with motivational interviewing. Int J Behav Nutr Phys Act 2012;9:18.

30. Deci EL, Ryan RM. The "What" and "Why" of goal pursuits: human needs and the self-determination of behavior. Psychol Inq 2000;11:227-68

31. Ryan RM, Patrick H, Deci EL, et al. Facilitating health behaviour change and its maintenance: interventions based on selfdetermination theory. The European Health Psychologist 2008;10.

32. $\mathrm{Ng} \mathrm{JY}$, Ntoumanis $\mathrm{N}$, Thøgersen-Ntoumani C, et al. selfdetermination theory applied to health contexts: a meta-analysis. Perspect Psychol Sci 2012;7:325-40.

33. Miller WR, Rollnick S. Meeting in the middle: motivational interviewing and self-determination theory. Int J Behav Nutr Phys Act 2012;9:25.

34. Lundahl B, Moleni T, Burke BL, et al. Motivational interviewing in medical care settings: a systematic review and meta-analysis of randomized controlled trials. Patient Educ Couns 2013;93:157-68.

35. Benzo R, Vickers K, Novotny PJ, et al. Health Coaching and chronic obstructive pulmonary disease rehospitalization. A Randomized Study. Am J Respir Crit Care Med 2016;194:672-80.

36. Härter M, Dirmaier J, Dwinger S, et al. Effectiveness of telephonebased health coaching for patients with chronic conditions: a randomised controlled trial. PLoS One 2016;11:e0161269.

37. Masterson Creber R, Patey M, Lee CS, et al. Motivational interviewing to improve self-care for patients with chronic heart failure: miti-hf randomized controlled trial. Patient Educ Couns 2016;99:256-64.

38. Moullec G, Lavoie KL, Rabhi K, et al. Effect of an integrated care programme on re-hospitalization of patients with chronic obstructive pulmonary disease. Respirology 2012;17:707-14.

39. Markland D, Ryan RM, Tobin VJ, et al. Motivational interviewing and self-determination theory. J Soc Clin Psychol 2005;24:811-31.

40. Rollnick S, Miller WR, Butler CC. Motivational interviewing in health care - Helping patients Change Behavior. New York: The Guilford Press, 2008.

41. Hoffmann TC, Glasziou PP, Boutron I, et al. Better reporting of interventions: template for intervention description and replication (TIDieR) checklist and guide. BMJ 2014;348:g1687.

42. MacCoun R, Perlmutter S. Blind analysis: hide results to seek the truth. Nature 2015;526:187-9.

43. Bellg AJ, Borrelli B, Resnick B, et al. Enhancing treatment fidelity in health behavior change studies: best practices and recommendations from the NIH behavior change consortium. Health Psychol 2004;23:443-51. 
44. Parry C, Mahoney E, Chalmers SA, et al. Assessing the quality of transitional care: further applications of the care transitions measure. Med Care 2008;46:317-22.

45. Hibbard JH, Mahoney ER, Stockard J, et al. Development and testing of a short form of the patient activation measure. Health Serv Res 2005;40(6 Pt 1):1918-30.

46. Herdman M, Gudex C, Lloyd A, et al. Development and preliminary testing of the new five-level version of EQ-5D (EQ-5D-5L). Qual Life Res 2011;20:1727-36.

47. Chen B, Vansteenkiste M, Beyers W, et al. Basic psychological need satisfaction, need frustration, and need strength across four cultures. Motiv Emot 2015;39:216-36.

48. Kroenke K, Spitzer RL, Williams JB, et al. The Patient Health Questionnaire Somatic, Anxiety, and Depressive Symptom Scales: a systematic review. Gen Hosp Psychiatry 2010;32:345-59.
49. Kroenke K, Spitzer RL, Williams JB. The PHQ-9: validity of a brief depression severity measure. J Gen Intern Med 2001;16:606-13.

50. Morisky DE, Green LW, Levine DM. Concurrent and predictive validity of a self-reported measure of medication adherence. Med Care 1986;24:67-74.

51. Diaz FJ. Measuring the individual benefit of a medical or behavioral treatment using generalized linear mixed-effects models. Stat Med 2016;35:4077-92.

52. World Medical Association Declaration of Helsinki (WMA). Ethical principles for medical research involving human subjects: 59th WMA general assembly, Seoul. 2008. http://www.wma.net/en/20activities/ 20humanrights/index.html (Retrieved 23 June 2016).

53. All European Academies (ALLEA) PWGoSaE. Statement on ethics education in science. 2013. http://www.allea.org/Content/ALLEA/ Statement Ethics Edu web final_2013 10_10.pdf (Retrieved 23 June 2016). 